\title{
Water in Comets: Observations and Models
}

\author{
Jacques Crovisier \\ Observatoire de Paris, F-92195 Meudon, France
}

\begin{abstract}
Cometary water, the main constituent of nucleus ices, is difficult to observe directly. Breakthroughs were made recently using new ground based infrared instruments and space techniques (ISO and SWAS) on comets C/1996 B2 (Hyakutake), C/1995 O1 (Hale-Bopp), 103P/Hartley 2, and C/1999 H1 (Lee): observations of water hot bands, of water rotational lines, and measurements of the $[\mathrm{D}] /[\mathrm{H}]$ ratio and orthoto-para ratio of water. We discuss the laboratory data necessary to model the water spectrum and to interpret these observations.
\end{abstract}

\section{Introduction}

Water is believed to be the main constituent of ices in cometary nuclei. Its sublimation is driving cometary activity at short distances $\left(r_{h}<4\right.$ AU) from the Sun. At $\approx 1 \mathrm{AU}$ from the Sun, the production of water ranges from $10^{28}$ molec. $\mathrm{s}^{-1}\left(300 \mathrm{~kg} \mathrm{~s}^{-1}\right)$ for a small comet like $46 \mathrm{P} /$ Wirtanen to $10^{31} \mathrm{molec}$. $\mathrm{s}^{-1}\left(300\right.$ ton $\left.\mathrm{s}^{-1}\right)$ for a giant comet like $\mathrm{C} / 1995 \mathrm{O} 1$ (Hale-Bopp). Cometary atmospheres are supersonic jets expanding from the nucleus with velocities 0.7 $1.5 \mathrm{~km} \mathrm{~s}^{-1}$ and temperatures $10-100 \mathrm{~K}$, depending upon the distance to Sun and gas production rate.

Despite its prevalent abundance, cometary water is difficult to study because it is practically impossible to observe from the ground. Recently, comets C/1996 B2 (Hyakutake) and C/1995 O1 (Hale-Bopp) offered opportunities to observe exceptionally bright comets with modern instrumentation and to gain new insights into the physics and chemistry of these bodies (Crovisier 1998; Irvine et al. 2000).

\section{Observations}

\subsection{Indirect observations of water}

This can be done by observing the products of water photolysis with a wealth of means: the $\mathrm{OH}$ radical in the radio (ground) or near-UV (ground and space) domains, atomic $\mathrm{O}$ (prompt emission of forbidden lines in the visible), atomic $\mathrm{H}$ (UV and visible). One needs a good knowledge of the water photolysis process: rates, branching ratios to various products, excitation state of the products (Crovisier 1989). This is now fairly well understood and there is an overall agreement between all methods, when consistent model parameters are adopted. 


\subsection{Direct observations of water}

Radio observations The possible detection of the $22 \mathrm{GHz}$ line from the ground is a debated topic (Bird et al. 1999; Graham et al. 2000); HDO was firmly observed through a submillimetre line in comets Hyakutake and Hale-Bopp (Bockelée-Morvan et al. 1998; Meier et al. 1998). The $1_{10}-1_{01}$ fundamental rotational line at $557 \mathrm{GHz}$ was detected for the first time from space with the Submillimeter Wave Astronomy Satellite (SWAS) in comet C/1999 H1 (Lee) (Neufeld et al. 2000). This is promising for future observations with ODIN and FIRST. Aboard ROSETTA, a microwave instrument (MIRO) will be mainly devoted to the in situ observation of this line in $\mathrm{H}_{2}{ }^{16} \mathrm{O}, \mathrm{H}_{2}{ }^{17} \mathrm{O}$ and $\mathrm{H}_{2}{ }^{18} \mathrm{O}$.

Infrared observations First direct detections of water were made in comet Halley from a stratospheric aircraft (KAO) or from space (VEGA/IKS). Hot bands of water (such as $\nu_{1}+\nu_{2}+\nu_{3}-\nu_{1}$ at $1.94 \mu \mathrm{m}$ or $\nu_{1}-\nu_{2}$ and $\nu_{3}-\nu_{2}$ at $4.7 \mu \mathrm{m}$ ) can now be observed from the ground using new-generation, high-resolution infrared spectrometers (Dello Russo et al. 2000; Weaver et al. 1999). The Infrared Space Observatory (ISO) could observe the water bands in the 2.7 and $6.7 \mu \mathrm{m}$ regions in comets Hale-Bopp and 103P/Hartley 2, as well as several rotation lines around $180 \mu \mathrm{m}$ (Crovisier et al. 1997, 1999).

\section{Modelling and results}

\subsection{Modelling water fluorescence}

Good models of the emission of water in comets are necessary to understand the excitation and physical conditions of the cometary environment. This modelling is crucial for reliable determinations of the water production rates from observations of hot bands or of a single rotational or rovibrational line.

The main emission mechanism is fluorescence induced by vibrational excitation by the solar infrared radiation. The resonant fluorescence of the fundamental bands of vibration is dominant in first approximation. However, in order to understand the hot bands that are now observed, it is necessary to consider the whole fluorescence pattern following excitation of high vibrational levels. Line strengths are lacking for some of the relevant hot bands in the HITRAN and GEISA data banks. A significant progress in this topic is the very comprehensive data base of Partridge \& Schwenke (1997).

The rotational distribution of water is largely controlled by collisions with neutrals in the inner coma, with ions in the outer coma. Collision cross-sections are required to assess this phenomenon. For a long time, only global crosssections (i.e., irrespective of the rotational level and collision-induced transition) were used in models (Bockelée-Morvan 1987). For $\mathrm{H}_{2} \mathrm{O}-\mathrm{H}_{2} \mathrm{O}$ collisions (which are presumably the dominant phenomenon), cross-sections were recently evaluated by Buffa et al. (2000) and will allow to achieve more realistic models. Other tricky points are the role of optical depth effects and the coupling ketween water excitation and radiative cooling in cometary atmospheres. 


\subsection{Deuterium in cometary water}

A crucial test for scenarios of comet formation is the abundance of deuterium. The $[\mathrm{D}] /[\mathrm{H}]$ ratio in cometary water is observed to be $3 \times 10^{-4}$, (BockeléeMorvan et al. 1998; Meier et al. 1998; Meier \& Owen 1999) which is 10 times larger than the protosolar value. This suggests that cometary water is coming from little-processed, deuterium-enriched protosolar water.

This ratio is also twice that observed for Earth ocean water. Earth water is thus not (all) coming from comets. However, one should note that $[\mathrm{D}] /[\mathrm{H}]$ has been measured in only three comets, all coming from the Oort cloud. Nothing is known about Jupiter-family comets, which experienced a different formation scenario, and which could have had a high impact rate on the Earth in the past.

\subsection{The ortho-to-para ratio of cometary water}

Water exists in ortho and para states which are not connected by radiative or collisional transitions. The ortho-to-para ratio and the corresponding spin temperature, which can be evaluated from water infrared spectra, may thus contain a primordial message (Mumma et al. 1987). A cold spin temperature $(\approx 30 \mathrm{~K})$ was determined from the ISO spectra of comets Hale-Bopp and 103P/Hartley 2 (Crovisier et al. 1997, 1999). This agrees with a previous determination in comet Halley. What is its meaning? It could be the temperature of formation of water (on grain surface); or due to re-equilibration in the cometary nucleus; or due to fractionation processes when water condensed. Laboratory data are clearly needed to understand these observations.

\section{References}

Bird, M.K., Janardhan, P., Wilson, T.L., et al. 1999, Earth Moon Plan., 78, 21 Bockelée-Morvan, D. 1987, A\&A, 181, 169

Bockelée-Morvan, D., Gautier, D., Lis, D.C., et al. 1998, Icarus, 133, 147

Buffa, G., Tarrini, O., Scappini, F., \& Cecchi-Pestellini, C. 2000, ApJS, 128, 597 Crovisier, J. 1989, A\&A, 213, 459

Crovisier, J. 1998, Faraday Discuss., 109, 437

Crovisier, J., Encrenaz, T., Lellouch, E., et al. 1999, ESA SP-427, 161

Crovisier, J., Leech, K., Bockelée-Morvan, D., et al. 1997, Science, 275, 1904

Dello Russo, N., Mumma, M.J., DiSanti, M.A., et al. 2000, Icarus, 143, 324

Graham, A.P., Butler, B.J., Kogan, L., et al. 2000, AJ, 119, 2465

Irvine, W., Schloerb, F.P., Crovisier, J., et al. 2000, in Protostars and Planets IV, ed. V. Mannings, A. Boss \& S. Russell (Univ. Arizona Press), 1159

Meier, R., Owen, T.C., Matthews, H.E., et al. 1998, Science, 279, 842

Meier, R., \& Owen, T.C. 1999, Space Scie. Rev., 90, 33

Mumma, M.J., Weaver, H.A., \& Larson, H.P. 1987, A\&A, 187, 419

Neufeld, D.A., Stauffer, J.R., Bergin, E.A., et al. 2000, AJ, 539, L151

Partridge, A., \& Schwenke, D.W. 1997, J. Chem. Phys., 106, 4618

Weaver, H.A., Brooke, T.Y., Chin, G., et al. 1999, Earth Moon Plan., 78, 71 